\title{
Treatment selection for patients with equivocal HER2 status and in luminal versus HER2-enriched disease
}

\author{
Giuseppe Vialea,b,*,† and Elisabetta Munzone ${ }^{\mathrm{c}}$ \\ ${ }^{a}$ University of Milan, Department of Oncology and Hemato-Oncology, Milan, Italy \\ ${ }^{b}$ Department of Pathology and Laboratory Medicine IEO, European Institute of Oncology IRCCS, Milan, Italy \\ ${ }^{c}$ Division of Medical Senology IEO, European Institute of Oncology IRCCS, Milan, Italy
}

\section{K E Y W O R D S}

HER2-positive breast cancer

HER2-targeted therapy

Neo-adjuvant therapy

CDK4/6 pathway

Immunotherapy

\begin{abstract}
A B S T R A C T
Equivocal HER2 status has been variably defined in the past, and its clinical implications have long been debated. In the 2018 focused update, ASCO/CAP guidelines recommended that tumours with double-equivocal (by immunohistochemistry and in situ hybridization assays) HER2 status should be considered HER2-negative due to the lack of evidence for any benefit of HER2-targeted therapy.

The biology and the response to systemic therapies of tumours co-expressing HR and HER2 is quite complex. There is an extensive bi-directional cross-talk between these 2 pathways, that may result in both intrinsic and acquired resistance to endocrine agents, as well as in lower sensitivity to HER2-targeted therapies. In fact, neoadjuvant studies indicate that pCR rates are significantly lower in HER2-positive/ER-positive than ER-negative tumours, regardless the type of HER2 targeted treatment.

The recent identification of different subtypes of HER2-positive breast cancer, according to the co-expression of HR and/or the molecular (intrinsic) subtyping, has prompted a renewed interest for clinical studies aimed at better tailoring the systemic therapy for these patients. A subgroup of them might not need chemotherapy if treated with dual HER2 blockade, and this option has been tested in a number of neo-adjuvant trials. In addition, triple targeting of HR, HER2, and CDK4/6 pathways simultaneously may be an effective treatment and overcome the drug resistance mechanisms that are typical of the disease.

Finally, HER2-positive breast cancer may well benefit from immunotherapeutic interventions with antiprogrammed cell death protein 1 (PD-1) and programmed cell death ligand 1 (PD-L1) agents.
\end{abstract}

(C) 2019 Elsevier Ltd. All rights reserved.

\section{Highlights}

- Patients with equivocal HER2 status do not benefit from HER2targeted therapy

- $\quad$ PCR rates after neoadjuvant chemotherapy are lower in HER2positive/ER-positive tumours

- Triple targeting of HR, HER2, and CDK4/6 or PD-1/PD-L1 may be an effective therapeutic strategy

\section{The challenges of equivocal HER2 status}

Expression and amplification of the HER2 gene in breast carcinoma are continuous variables, making it arbitrary to define precise thresholds for positive and negative results. Accordingly, for both

${ }^{*}$ Corresponding author at: Department of Pathology and Laboratory Medicine, Istituto Europeo di Oncologia, Via Ripamonti 435-20141 Milano, Italy.

${ }^{\dagger}$ E-mail address: giuseppe.viale@ieo.it (G. Viale).

This article was published as part of a supplement sponsored by St. Gallen Oncology Conferences. immunohistochemical (IHC) and in situ hybridization (ISH) assays we have been forced to accept a grey zone of equivocal results between those that are frankly positive and frankly negative.

In the IHC assays, equivocal results are characterised by a weak to moderate circumferential membrane staining observed in more than $10 \%$ invasive tumour cells. Less common equivocal patterns of staining include an intense but incomplete membrane staining in more than $10 \%$ invasive tumour cells (most often encountered in invasive micropapillary carcinomas), and an intense circumferential membrane staining in $10 \%$ or less tumour cells. An equivocal IHC result requires reflex testing of the same sample with ISH assays, to assess whether or not the HER2 gene is amplified [1].

In the ISH assays, equivocal results have been variably defined in the past, according to a ratio (between the mean gene copy number and the chromosome enumeration probe 17 ) of 1.8 to 2.2 , or to a mean gene copy number of 4 to $<6$ in tumours with a ratio of $<2[2,3]$.

The clinical implications of "double-equivocal" (i.e.: with equivocal results both in IHC and ISH assays) HER2 status of breast carcinomas have long been debated and have challenged oncologists and patients due to a perceived ambivalence about whether to recommend HER2targeted therapy. In 2013, the ASCO/CAP guideline recommendations 
suggested that oncologists might consider HER2-targeted therapy in such cases on the basis of patient status (comorbidities, prognosis, and so on) and patient preferences, after discussing available clinical evidence [3]. In the 2018 focused update of the same guidelines, however, it is now recommended that these cases be considered HER2-negative (without additional testing on the same specimen), due to the lack of evidence for any benefit from HER2-targeted therapy. Clinical correlation with other factors in a particular case (such as grade and special histologic subtypes) or repeat testing of other tissue samples (of the primary tumours or lymph node metastasis if present) from the patient may also be appropriate in this setting. In particularly challenging cases or if the results are in question, expert consultation may be appropriate and may include alternative probes or other molecular testing [1]. The vast majority of these double-equivocal tumours have low abundance of HER2 mRNA and are molecularly classified as luminal subtypes (discussed below).

\section{Intratumoral heterogeneity of HER2-positive breast carcinomas}

HER2-positive breast cancer is defined as a tumour overexpressing the HER2 protein (with an immunohistochemical score of $3+$ ) and/or showing gene amplification (with a ratio between the mean gene copy number and the chromosome enumeration probe 17 of $\geq 2$, or a mean gene copy number of $\geq 6$ ) [1].

Though the vast majority of HER2-positive breast cancers exhibit overexpression of the protein and/or gene amplification in almost all the neoplastic cells, still a number of cases show intratumoral heterogeneity, with an intermingling of positive and negative populations of invasive tumour cells. Sometimes, the heterogeneity correlates with, and it may be predicted by, a morphological heterogeneity of the tumour, like in case of mixed histologies (e.g.: ductal and lobular) or of tumour areas with different histological grades. More often, however, there are no identifiable morphological differences between the HER2-positive and the HER2-negative components. The current guidelines recommend that tumours are considered HER2-positive when more than $10 \%$ of the invasive neoplastic cells show overexpression or amplification of the HER2 gene. These features, however, should be observed within a homogeneous and contiguous population of invasive tumour cells [1].

\section{Co-expression of hormone receptors in HER2-positive tumours}

The prototypical features of HER2-positive breast carcinomas include lack of hormone receptor (HR) expression, ductal histotype, moderate to high tumour grade, and high Ki67 labelling index [4,5]. Actually, however, $60 \%$ or more of HER2-positive breast cancers co-express estrogen receptors (ER) and $40 \%$ to $50 \%$ of them also express progesterone receptors (PgR), being often referred to as "triplepositive" breast cancer [6,7]. Co-expression of HR may be observed in tumours with morphologically different cell populations (e.g.: mixed ductal and lobular carcinomas, or tumours with high- and low-grade components), where the ductal and/or the high-grade component is more likely to be HER2-positive, and the other component HRpositive and HER2-negative. In most instances, however, expression of HR together with over-expression and/or amplification of HER2 are exhibited by the very same tumour cells. In the majority of these cases, the neoplastic population is homogeneously positive for HER2, and the co-expression of HR is restricted to a subset of the tumour cells. In particular, ER immunoreactivity is more often encountered in a lower percentage of cells, and the staining intensity is weaker, than in HER2-negative tumours. PgR immunoreactivity may overlap ER expression, or be lower or absent [6]. Less commonly, tumours may show HER2 overexpression/amplification and ER immunoreactivity (with or without PgR) in the vast majority -if not all- the neoplastic cells.

\section{Molecular classification of HER2-positive breast cancer}

The technology of gene expression profiling has unveiled the striking molecular heterogeneity of breast carcinomas, and has paved the way for a new classification of these tumours, based on their molecular characteristics (intrinsic subtypes). Four major molecular classes (Luminal A, Luminal B, HER2-enriched and Basal-like) have been identified, and the prognostic value of this classification has been repeatedly confirmed $[8,9]$. The molecular classes do partially overlap, but do not coincide with, those defined by immunohistochemical surrogates (ER, PgR, Ki67 and HER2). This holds also true for tumours that are clinically HER2-positive, based on IHC and ISH assays. When subjected to gene expression profiling, only approximately half of these tumours belong to the HER2-enriched class, while the remaining cluster with the Luminal A and B, or the Basallike subtypes [10].

It is tempting to speculate that the molecularly HER2-enriched tumours are those clinically HER2-positive but HR-negative, whereas the tumours that co-express HR would correspond to those that are molecularly classified as Luminal. Unfortunately, however, this assumption has not been confirmed by studies comparing intrinsic subtypes and the IHC surrogates. Indeed, a fraction (10\% to $18 \%$ ) of tumours that are clinically HER2-positive and HR-negative belong to the Luminal intrinsic subtype, and $43 \%$ to $54 \%$ of tumours coexpressing HR belong to the HER2-enriched subtype when tested with the PAM50 assay [10-13].

We also have compared the clinical and molecular classification of 5788 tumour samples of patients enrolled in the MINDACT trial using an alternative multigene expression panel (TargetPrint, Agendia, The Netherlands). Of the 429 clinically HER2-positive and ER-positive samples, 216 (50.3\%) were classified as HER2-enriched by the molecular assay [14].

\section{Biological and clinical implications of hormone receptor co- expression and of intrinsic subtyping}

HER2-positive breast cancers that do not co-express HR, mostly belonging to the HER2-enriched intrinsic subtype, are driven by the activation of the HER2 pathway (HER2 addiction), and are exquisitely sensitive to HER2-targeted therapies.

On the other hand, the biology and the response to systemic therapies of tumours co-expressing HR is much more complex. They may be oncogenically driven by either or both the HR and the HER2 signalling pathways, in a similar manner as for a hybrid car with two different engines. There is an extensive bi-directional cross-talk between these two pathways, that may result in both intrinsic and acquired resistance to endocrine agents, as well as in lower sensitivity to HER2-targeted therapies. There is plenty of evidence from neo-adjuvant studies that PCR rates are significantly lower in ER-positive than ER-negative tumours, regardless the type of HER2targeted treatment [7], either trastuzumab alone or the dual blockade with trastuzumab and pertuzumab [15] or with trastuzumab and lapatinib [16].

Moreover, the pooled analysis of the German neo-adjuvant studies suggests that PCR may be a suitable surrogate endpoint for HER2positive/HR-negative but not for HER2-positive/HR-positive breast cancer patients [17].

In the adjuvant setting, however, a significant interaction between HR status (or intrinsic subtypes) and the treatment effect of single agent trastuzumab or of dual HER2 blockade (trastuzumab plus lapatinib or pertuzumab) has not been documented. At variance with the neo-adjuvant setting, patients enrolled in adjuvant trials have also received endocrine treatment, and the prevalent mechanism(s) of action of HER2-targeted agents may be different in the two settings. Nevertheless, there are some limitations in the studies, such 
as the simplistic definition of HR status ( positive vs. negative), which does not take into account the extent of HR expression, and the lack of centralized evaluation.

In a retrospective evaluation of records from more than 3000 patients enrolled in the HERA trial, however, we reported for the first time a significant association between ER-positive tumours with lower levels of HER2 gene amplification and less magnitude of benefit to adjuvant trastuzumab [18].

Recently, in the post-neoadjuvant setting, results from the KATHERINE trial indicate that among patients with HER2-positive early breast cancer who had residual invasive disease after completion of neoadjuvant therapy, the risk of recurrence of invasive breast cancer or death was $50 \%$ lower with adjuvant T-DM1 than with trastuzumab alone, irrespective of HR status [19].

Prior to this, however, the ExeNET trial showed that 1 year of neratinib (an irreversible pan-HER tyrosine kinase inhibitor) after chemotherapy and trastuzumab adjuvant therapy significantly reduces the likelihood of clinically relevant breast cancer relapse [20]. Neratinib seemed to have a greater effect in patients with HRpositive breast cancer (most of whom were receiving concurrent hormone therapy) than in those with HR-negative disease. In a model of HER2-positive/ER-positive xenografts after tumour regrowth following treatment with trastuzumab and paclitaxel, mirroring the ExteNET population, treatment with neratinib and fulvestrant, compared with fulvestrant alone, showed prolonged complete responses in vivo and preserved sensitivity to endocrine therapy after recurrence [21]. This observation suggests an absence of crossresistance between trastuzumab and neratinib in the hormone receptor-positive population [20].

\section{New treatment options for patients with HER2-positive disease}

The identification of different subtypes of HER2-positive breast cancer, according to the co-expression of HR and/or the intrinsic subtyping, has prompted a renewed interest for clinical studies aimed at better tailoring the systemic therapy of the affected patients.

It has been mentioned already that the exquisite sensitivity of HER2-addicted tumours to targeted therapies suggests that a subgroup of patients with early-stage HER2-positive breast cancer, mostly those with the HER2-enriched subtype, might not need chemotherapy if treated with dual HER2 blockade. This option has been tested in a number of neo-adjuvant trials, where the combination of trastuzumab and lapatinib or pertuzumab without cytotoxic therapy has achieved remarkable pCR rates of approximately 20-30\% [12,15,22-25].

Neo-adjuvant regimens without use of systemic chemotherapy have also been exploited for HER2-positive/HR-positive disease. In the ADAPT HER2-positive/HR-positive trial, neo-adjuvant T-DM1 (with or without endocrine therapy) given for only 12 weeks achieved a meaningful pCR rate, thus sparing a substantial number of patients the adverse effects of systemic chemotherapy [26].

HER2-positive/HR-positive (or Luminal) tumours, however, represent a major therapeutic challenge because they harbour multiple oncogenic drivers and pathways of therapeutic resistance. The concomitant block of the HR pathway together with an effective block of homo- and hetero-dimer signalling of HER2 by trastuzumab and lapatinib or pertuzumab without addition of systemic chemotherapy has been tested in the neo-adjuvant and in the metastatic settings. The neoadjuvant TBCRC006 trial tested the hypothesis, and showed a relatively high rate $(21 \%)$ of pCR in breast with the combination of lapatinib, trastuzumab and letrozole [23]. In the phase II PerELISA trial, patients with HER2positive/HR-positive operable breast cancer received 2-weeks letrozole. Then patients classified as molecular responders (Ki67 relative reduction after 2 weeks $>20 \%$ from baseline) continued letrozole and started trastuzumab and pertuzumab for 5 cycles achieving pCR in $20.6 \%$ of the cases. pCR rate, however, was significantly higher in HER2-enriched vs. other subtypes (45.5\% vs. 13.8\%) according to PAM50 [24].

In the PAMELA trial, investigating the role of lapatinib and trastuzumab without chemotherapy, the pCR rate (breast only) in the cohort of hormone receptor positive/HER2-enriched after endocrine therapy plus dual HER2 blockade was 32\% [12].

In the metastatic setting, dual block of HER2 with trastuzumab and pertuzumab combined with an aromatase inhibitor in the PERTAIN study led to a significant 3-months longer PFS than observed with trastuzumab and aromatase inhibitor [27]. These trials indicated that dual targeting of HER2 is superior to single block in interfering with the cross-talk between HR and HER2.

ER and HER2 signalling pathways exert a synergistic effect on the cell cycle checkpoints, and there are multiple feedback loops between these pathways. It is therefore attractive to investigate the combination of CDK4/6 inhibitors with HER2-targeted agents and endocrine interventions for the treatment of HER2-positive/ HR-positive disease. Triple targeting of HR, HER2, and CDK4/6 pathways simultaneously may be an effective treatment and overcome the drug resistance mechanisms that are typical of the disease. Furthermore, the combination is safe, with a favourable toxicity profile and non-overlapping side effects.

This therapeutic strategy has been successfully exploited in the neo-adjuvant setting [28] and in the advanced setting [29], and additional clinical trials testing the triple blockade of ER, HER2 and CDK4/6 in locally advanced and metastatic HER2positive/HR-positive breast cancer are currently recruiting patients (ClinicalTrials.gov identifier NCT03054363, NCT02947685, NCT02448420, NCT02675231 and NCT02657343).

An overlapping mechanism of resistance to all three drugs probably exists (cyclin E overexpression) [30,31]. The assessment of this and other potential resistance mechanisms should be performed in the ongoing clinical trials testing triple combinations of antihormonal therapy with HER2 and CDK4/6-targeted agents.

Based on non-overlapping resistance mechanisms, other possible combined therapies for the treatment of HR-positive and HER2positive breast cancer may be exploited (for example, a combination of anti-hormonal drugs with HER2-targeted agents and PI3 $\mathrm{K}$ or mTOR inhibitors). Some of these combinations are now being tested in a clinical trial in the metastatic setting (ClinicalTrials.gov identifier NCT03767335).

Finally, HER2-positive breast cancer may well benefit from immunotherapeutic interventions with anti-programmed cell death protein 1 (PD-1) and programmed cell death ligand 1 (PD-L1) agents. Indeed, these tumours may have substantial amounts of tumour infiltrating lymphocytes (TILs) that are associated with the likelihood of achieving a pathological complete response and having improved survival [32]. Preclinical studies have already suggested that the combination of trastuzumab with drugs targeting immune checkpoints could overcome trastuzumab resistance [33].

High expression of PD-1 and PD-L1, and other checkpoint molecules has been observed in immune cells of HER2-positive breast cancer [34]. We have already shown that the combination of an anti-PD-1 drug (pembrolizumab) and trastuzumab was safe and showed activity and durable clinical benefit in patients with PD-L1positive, trastuzumab-resistant, advanced, HER2-positive breast cancer [35]. A regimen including dual targeting of HER2 with trastuzumab and pertuzumab and an anti-check point (atezolizumab or pembrolizumab) agent is currently being tested in the neoadjuvant setting (APTneo trial, ClinicalTrials.gov identifier NCT03125928 and neoHIP trial NCT03747120). 


\section{Epilogue}

HER2-positive breast cancer is a biologically and clinically heterogeneous disease, as documented by pathology-based assessment of HR and HER2 status, and by the intrinsic subtyping derived from gene expression profiles. HER2-enriched tumours (most of which do not co-express hormone receptors) are exquisitely sensitive to HER2targeted agents, to the point that at least some patients may be cured by dual HER2 blockade only, without systemic chemotherapy. Tumours co-expressing HR (most of which belong to the luminal intrinsic subtypes), however, are relatively resistant to both endocrine interventions and HER2-targeted therapies, thus requiring a combination therapy aimed at blocking both signalling pathways. Addition of CDK4/6 inhibitors to endocrine and HER2-targeted treatments has recently shown promising results in the neo-adjuvant setting, and it is currently being tested in a number of ongoing clinical trials. Finally, there is initial evidence in the advanced setting that HER2-positive breast cancer may benefit from the combination of HER2-targeted agents and immunotherapy with anti-PD-1 or PD-L1 agents, and this therapeutic strategy is also being investigated in the neo-adjuvant setting.

\section{Conflict of interest statement}

The authors declare no conflict of interest.

\section{Funding source}

None.

\section{References}

[1] Wolff AC, Hammond MEH, Allison KH, et al. Human epidermal growth factor receptor 2 testing in breast cancer: American Society of Clinical Oncology/College of American Pathologists clinical practice guideline focused update. J Clin Oncol 2018;36:2105-22.

2] Wolff AC, Hammond ME, Schwartz JN, et al. American Society of Clinical Oncology/ College of American Pathologists guideline recommendations for human epidermal growth factor receptor 2 testing in breast cancer. J Clin Oncol 2007;25: 118-45.

[3] Wolff AC, Hammond ME, Hicks DG, et al. Recommendations for human epidermal growth factor receptor 2 testing in breast cancer: American Society of Clinical Oncology/College of American Pathologists clinical practice guideline update. J Clin Oncol 2013;31:3997-4013.

[4] Slamon DJ, Clark GM, Wong SG, et al. Human breast cancer: Correlation of relapse and survival with amplification of the HER-2/neu oncogene. Science 1987;235:177-82.

[5] Slamon DJ, Godolphin W, Jones LA, et al. Studies of the HER-2/neu proto-oncogene in human breast and ovarian cancer. Science 1989;244:707-12.

[6] Lal P, Tan LK, Chen B. Correlation of HER-2 status with estrogen and progesterone receptors and histologic features in 3655 invasive breast carcinomas. Am J Clin Pathol 2005; 123:541-6.

[7] Vici P, Pizzuti L, Natoli C, et al. Triple positive breast cancer: A distinct subtype? Cancer Treat Rev 2015;41:69-76.

[8] Perou CM, Sorlie T, Eisen MB, et al. Molecular portraits of human breast tumours. Nature. 2000;406:747-52.

[9] Prat A, Parker JS, Fan C, Perou CM. PAM50 assay and the three-gene model for identifying the major and clinically relevant molecular subtypes of breast cancer. Breast Cancer Res Treat 2012;135:301-6.

[10] Bastien RRL, Rodríguez-Lescure A, Ebbert MTW, et al. PAM50 breast cancer subtyping by RT-qPCR and concordance with standard clinical molecular markers. BMC Medical Genomics 2012;5:44.

[11] Cheang MCU, Martin M, Nielsen TO, et al. Defining breast cancer intrinsic subtypes by quantitative receptor expression. Oncologist 2015;20:474-82.

[12] Llombart-Cussac A, Cortés J, Paré L, et al. HER2-enriched subtype as a predictor of pathological complete response following trastuzumab and lapatinib without chemotherapy in early-stage HER2-positive breast cancer (PAMELA): An open-label, single-group, multicentre, phase 2 trial. Lancet Oncol 2017; 18:545-54.

[13] Kyung Kim HK, Hee Park KH, Kim Y, et al. Discordance of the PAM50 intrinsic subtypes compared with IHC-based surrogate in breast cancer patients: Potential implication of genomic alterations of discordance. Cancer Res Treat 2019. doi: 10.4143/crt.2018.342. [Epub ahead of print].

[14] Viale G, Slaets L, de Snoo FA, et al. Discordant assessment of tumor biomarkers by histopathological and molecular assays in the EORTC randomized controlled10041/BIC
03-04 MINDACT trial breast cancer intratumoral heterogeneity and DCIS or normal tissue components are unlikely to be the cause of discordance. Breast Cancer Res Treat 2016;155:463-9.

[15] Gianni L, Pienkowski T, Im YH, et al. Efficacy and safety of neoadjuvant pertuzumab and trastuzumab in women with locally advanced, inflammatory, or early HER2-positive breast cancer (NeoSphere): A randomised multicentre, open-label, phase 2 trial. Lancet Oncol 2012;13:25-32.

[16] Baselga J, Bradbury I, Eidtmann H, et al. Lapatinib with trastuzumab for HER2-positive early breast cancer (NeoALTTO): A randomised, open-label, multicentre, phase 3 trial. Lancet 2012;379:633-40.

[17] von Minckwitz G, Untch M, Blohmer JU, et al. Definition and impact of pathologic complete response on prognosis after neoadjuvant chemotherapy in various intrinsic breast cancer subtypes. J Clin Oncol 2012;30:1796-804.

[18] Loi S, Dafni U, Karlis D, et al. Effects of estrogen receptor and human epidermal growth factor receptor-2 levels on the efficacy of trastuzumab: A secondary analysis of the HERA trial. JAMA Oncol 2016;2:1040-7.

[19] von Minckwitz G, Huang CS, Mano MS, et al. Trastuzumab emtansine for residual invasive HER2-positive breast cancer. N Engl J Med 2019;380:617-28.

[20] Martin M, Holmes FA, Ejlertsen B, et al. Neratinib after trastuzumab-based adjuvant therapy in HER2-positive breast cancer (ExteNET): 5-year analysis of a randomised, double-blind, placebo-controlled, phase 3 trial. Lancet Oncol 2017;18: $1688-700$.

[21] Schwarz LJ, Croessmann SE, Avogadri-Connors F, et al. Neratinib + fulvestrant enhances antitumor activity in a HER2+/estrogen receptor (ER)+ breast cancer xenograft model after trastuzumab-based therapy. Cancer Res 2017;77:4818(abstr).

[22] Prat A, Baselga J. Dual human epidermal growth factor receptor 2 (HER2) blockade and hormonal therapy for the treatment of primary HER2-positive breast cancer: One more step toward chemotherapy-free therapy. J Clin Oncol 2013;31:1703-6.

[23] Rimawi MF, Mayer IA, Forero A, et al. Multicenter phase II study of neoadjuvant lapatinib and trastuzumab with hormonal therapy and without chemotherapy in patients with human epidermal growth factor receptor 2- overexpressing breast cancer: TBCRC 006. J Clin Oncol 2013;31:1726-31.

[24] Guarneri V, Dieci MV, Bisagni G, et al. De-escalated therapy for HR+/HER2+ breast cancer patients with Ki67 response after 2 weeks letrozole: Results of the PerELISA neoadjuvant study. Ann Oncol 2019. doi: 10.1093/annonc/mdz055. [Epub ahead of print].

[25] Veeraraghavan J, De Angelis C, Reis-Filho JS, et al. De-escalation of treatment in HER2positive breast cancer: Determinants of response and mechanisms of resistance. Breast 2017;34:S19-26.

[26] Harbeck N, Gluz O, Christgen M, et al. De-Escalation Strategies in Human Epidermal Growth Factor Receptor 2 (HER2)-positive early breast cancer (BC): Final analysis of the West German Study Group Adjuvant Dynamic Marker-Adjusted Personalized Therapy Trial optimizing risk assessment and therapy response prediction in early BC HER2- and hormone receptor-positive phase II randomized trial-Efficacy, safety, and predictive markers for 12 weeks of neoadjuvant Trastuzumab Emtansine with or without endocrine therapy (ET) versus Trastuzumab plus ET. J Clin Oncol 2017;35:3046-54.

[27] Rimawi M, Ferrero J-M, de la Haba-Rodriguez J, et al. First-Line trastuzumab plus an aromatase inhibitor, with or without pertuzumab, in human Epidermal Growth Factor Receptor 2-positive and hormone receptor-positive metastatic or locally advanced breast cancer (PERTAIN): A randomized, open-label phase II trial. J Clin Oncol 2018;36:2826-35.

[28] Gianni L, Bisagni G, Colleoni M, et al. Neoadjuvant treatment with trastuzumab and pertuzumab plus palbociclib and fulvestrant in HER2-positive, ER-positive breast cancer (NA-PHER2): An exploratory, open-label, phase 2 study. Lancet Oncol 2018;19:249-56.

[29] Villagrasa Gonzalez P, Prat A, Oliveira M, et al. 321TiPSOLTI-1303 PATRICIA: A phase II study of palbociclib and trastuzumab (with or without letrozole in ER+) in previously trastuzumab-pretreated, postmenopausal patients with HER2-positive metastatic breast cancer. Ann Oncol 2017;28. Doi: 10.1093/annonc/mdx365.084.

[30] Akli S, Bui T, Wingate H, et al. Low-molecular-weight cyclin E can bypass letrozoleinduced G1 arrest in human breast cancer cells and tumors. Clinical Cancer Res 2010;16:1179-90.

[31] Scaltriti M, Eichhorn PJ, Cortes J, et al. Cyclin E amplification/ overexpression is a mechanism of trastuzumab resistance in HER2+ breast cancer patients. Proc Natl Acad Sci USA 2011;108:3761-6.

[32] Savas P, Salgado R, Denkert C, et al. Clinical relevance of host immunity in breast cancer: From TILs to the clinic. Nat Rev Clin Oncol 2016;13:228-41.

[33] Stagg J, Loi S, Divisekera U, et al. Anti-ErbB-2 mAb therapy requires type I and II interferons and synergizes with anti-PD-1 or anti-CD137 mAb therapy. Proc Natl Acad Sci USA 2011;108:7142-7.

[34] Denkert C, von Minckwitz G, Brase JC, et al. Tumor-infiltrating lymphocytes and response to neoadjuvant chemotherapy with or without carboplatin in human epidermal growth factor receptor 2-positive and triple-negative primary breast cancers. J Clin Oncol 2015;33:983-91.

[35] Loi S, Giobbie-Hurder A, Gombos A, et al. Pembrolizumab plus trastuzumab in trastuzumab-resistant, advanced, HER2-positive breast cancer (PANACEA): A singlearm, multi-centre, phase 1b-2 trial. Lancet Oncol 2019. doi: 10.1016/S1470-2045(18) 30812-X. [Epub ahead of print]. 